Bull. Austral. Math. Soc.

$57 \mathrm{~N} 10,57 \mathrm{M} 05$

VOL. 58 (1998) [233-237]

\title{
NILPOTENT QUOTIENTS OF FUNDAMENTAL GROUPS OF SPECIAL 3-MANIFOLDS WITH BOUNDARY
}

\author{
Gabriela Putinar
}

We use a Betti number estimate of Freedman-Hain-Teichner to show that the maximal torsion-free nilpotent quotient of the fundamental group of a 3-manifold with boundary is either $\mathbf{Z}$ or $\mathbf{Z} \oplus \mathbf{Z}$. In particular we reobtain the Evans-Moser classification of 3-manifolds with boundary which have nilpotent fundamental groups.

I. Nilpotent quotients of groups are closely related to the rational homotopy type of $K(\pi, 1)$ spaces. The object which defines this relation is called a Malcev completion and is dual to the one-minimal model [5]. Because of its naturality and computability, the Malcev completion can be used to describe nilpotent quotients of $\pi_{1}(X)$ from homotopic information about $X$. This approach is adopted in [2], [6], to study nilpotent quotient groups of orientable closed 3-manifolds.

Two types of (closed, orientable) 3-manifolds are distinguished in [2]: the special type 3-manifolds have finite number of stages in their one-minimal models, or equivalently their rational lower central series stabilises, that is, they admit a maximal torsion-free nilpotent quotient. The other 3-manifolds are of general type. It is shown in [2] that for 3-manifolds of special type the only possible maximal torsion-free nilpotent quotient groups of the fundamental group are $1, \mathrm{Z}$ or the Heisenberg groups $H_{n}, n \in \mathrm{Z}$. By contrast [4] shows that in the general type case, the one-minimal models grow exponentially.

In this note we extend the special case to 3-manifolds with boundary. We find that the maximal torsion-free nilpotent quotient of the fundamental group of such a manifold must be $\mathbf{Z}$ or $\mathbf{Z} \oplus \mathbf{Z}$. If moreover the fundamental group itself is nilpotent, it follows that the 3-manifold is (essentially) either the solid torus or a thickened 2-torus.

II. Let us collect first a few definitions and facts about nilpotent groups and Malcev completions.

Let $G$ be a group. Its lower central series is a decreasing series defined inductively by $\Gamma_{2}=[G, G], \Gamma_{r+1}=\left[G, \Gamma_{r}\right]$, with $[$,$] denoting commutators. We say G$ is nilpotent (respectively, prenilpotent) if $\Gamma_{r}=1$ for some $r$ (respectively, if $\Gamma_{r}=\Gamma_{r+1}$ for some $r$ ). It follows that $G$ is prenilpotent if and only if it has a maximal nilpotent quotient.

Received 28th January, 1998

I wish to thank Michael Freedman for suggesting this question and for interesting discussions on the subject.

Copyright Clearance Centre, Inc. Serial-fee code: 0004-9729/98 \$A2.00+0.00. 
There are rational versions of the above definitions. The $r$-th term of the rational lower central series is defined by:

$$
\Gamma_{r} \mathbf{Q}=\operatorname{Rad}\left(\Gamma_{r}\right)=\left\{g \in G ; \exists n \in \mathbf{Z}, n \neq 0, g^{n} \in \Gamma_{r}\right\}
$$

It has the defining property that $G / \Gamma_{r}{ }^{\mathbf{Q}}=G / \Gamma_{r} /$ torsion.

We obtain the definition of rationally (pre) nilpotent groups by replacing $\Gamma_{r}$ with $\Gamma_{r} \mathbf{Q}$ in the above definitions. Note that $G$ is rationally prenilpotent if and only if $G$ has a maximal torsion-free nilpotent quotient.

We describe next the construction of the Malcev completion of a group $G$ using nilpotent quotients. Let $N_{r}=G / \Gamma_{r}$. We have a tower of nilpotent quotients of $G$ :

$$
\ldots \rightarrow N_{r} \rightarrow N_{r-1} \rightarrow \ldots \rightarrow N_{2} \rightarrow 0
$$

and at each stage, a central extension:

$$
0 \rightarrow \Gamma_{r-1} / \Gamma_{r} \rightarrow N_{r} \rightarrow N_{r-1} \rightarrow 0
$$

Central extensions are classified by elements $k_{r}$ in $H^{2}$ (base; fibre). The tower of extensions above can be tensored with $\mathbf{Q}$ inductively (starting with $N_{2} \otimes \mathrm{Q}$ which since $N_{2}$ is Abelian can be defined as the usual $\otimes \mathbf{Q}$ ) and such that the new invariants are precisely $k_{r} \otimes \mathbf{Q}$ in the corresponding $H^{2} \otimes \mathbf{Q}$.

This new, rationalised tower is called the Malcev completion of $G$, denoted $G \otimes \mathbf{Q}$. Viewed as a Postnikov tower, it leads to a one-minimal model as in [5].

If $G$ is nilpotent we have a natural embedding of groups $G /$ torsion $\longrightarrow G \otimes \mathbf{Q}$. Further $G \otimes \mathbf{Q}$ is uniquely divisible (that is, for every $x \in G \otimes \mathbf{Q}$ and every $n \in \mathbf{Z}, n \neq 0$, there exists a unique $y \in G \otimes \mathbf{Q}$ such that $x=y^{n}$ ), and is the minimal uniquely divisible group containing $G /$ tors.

In conclusion let us note that the above are related by : $G$ is rationally prenilpotent if and only if $G \otimes \mathrm{Q}$ is a finite stage tower if and only if $G$ has a finite-stage one-minimal model.

III. With the above definitions, a (connected) 3-manifold is called of special type if its fundamental group is rationally prenilpotent, that is, if it admits a maximal torsion-free nilpotent quotient.

Let $M$ be a compact, oriented 3-manifold with boundary. In order to have a genuinely non-empty boundary we shall assume $\partial(\widehat{M}) \neq \emptyset$, where $\widehat{M}$ is obtained from $M$ by capping-off all the $S^{2}$-boundary components with 3-cells. This is equivalent to assuming that $M$ itself has at least one boundary component which is not a sphere. Also, replacing $M$ with $\widehat{M}$ does not change the fundamental group.

The classification in the boundary case is given by: 
THEOREM 1. Let $M$ be a compact orientable 3-manifold such that $\widehat{M}$ has nonempty boundary. If $M$ is of special type then the maximal torsion-free nilpotent quotient of $\pi_{1}(M)$ is $\mathbf{Z}$ or $\mathbf{Z} \oplus \mathbf{Z}$.

For the proof we use the following Betti numbers estimate, which, together with a stronger version, is the central result of [2]:

THEOREM 2. [2]. Let $G$ be a nilpotent group with $b_{1}(G)<\infty$. Then either $G \otimes \mathbf{Q}=1, \mathbf{Q}, \mathbf{Q} \oplus \mathbf{Q}$, or $b_{2}(G)>b_{1}(G)^{2 / 4}$.

Here $G \otimes \mathbf{Q}$ is the Malcev completion of $G$, while $b_{i}(G)$ denote the rational Betti numbers of $G$.

We shall also use Stallings' exact sequence from homological algebra, which says:

If $N$ is a normal subgroup of a group $G$, and if $Q=G / N$ is the quotient group, then there is a natural exact sequence:

$$
H_{2}(G) \rightarrow H_{2}(Q) \rightarrow N /[G, N] \rightarrow H_{1}(G) \rightarrow H_{1}(Q) \rightarrow 0 .
$$

Proof of Theorem 1: Let $M$ be special, let $G=\pi_{1}(M) / \Gamma_{r} \mathbf{Q}$ be the maximal torsion-free nilpotent quotient, with notation as in II. By maximality, $\Gamma_{r} \mathbf{Q}=\Gamma_{r+1}{ }^{\mathbf{Q}}$.

Since we may assume $M=\widehat{M}$, we have $g_{i} \geqslant 1$ for $1 \leqslant i \leqslant n$, where $g_{i}$ denotes the genus of the $i$-th boundary component and $n$ is the number of components of $M$. By Poincaré duality we have:

$$
\chi(M)=\frac{1}{2} \chi(\partial M)=\sum_{i=1}^{n}\left(1-g_{i}\right) \leqslant 0,
$$

which implies $b_{1}(M) \geqslant b_{2}(M)+1$.

We claim that also $b_{1}(G) \geqslant b_{2}(G)+1$. (Note that this will imply $G \neq 0$ ). Since $b_{1}(G)=b_{1}\left(\pi_{1}(M)\right)=b_{1}(M)$, it suffices to show $b_{2}(M) \geqslant b_{2}(G)$. Indeed, $H_{2}(M) \rightarrow$ $H_{2}\left(\pi_{1}(M)\right)$ is always onto, while

$$
H_{2}\left(\pi_{1}(M)\right) \longrightarrow H_{2}(G) \longrightarrow \Gamma_{r}^{\mathbf{Q}} / \Gamma_{r+1}^{\mathbf{Q}}=0
$$

is exact by Stallings' exact sequence, tensored with $\mathbf{Q}$.

Next we apply Theorem 2 to the group $G$. Assuming $G \otimes \mathbf{Q} \neq \mathbf{Q}$ and $G \otimes \mathbf{Q} \neq \mathbf{Q} \oplus \mathbf{Q}$, from the above we obtain:

$$
b_{2}(G)>\frac{1}{4} b_{1}(G)^{2} \geqslant \frac{1}{4}\left(b_{2}(G)+1\right)^{2},
$$

a contradiction. So $G \otimes \mathbf{Q}=\mathbf{Q}$ or $\mathbf{Q} \oplus \mathbf{Q}$. Finally this implies $G=\mathbf{Z}$ or $G=\mathbf{Z} \oplus \mathbf{Z}$ : Indeed, since $G$ is nilpotent, torsion free, the natural map $G \rightarrow G \otimes \mathbf{Q}$ is an embedding. 
Because $G \otimes \mathbf{Q}=\mathbf{Q}^{k}(\mathrm{k}=1,2)$ is Abelian and $G$ is finitely-generated, it follows that $G=Z^{l}$ for some $\ell$. But $l=k$ by the minimality of the Malcev completion.

IV. As a particular case we reobtain the classification [1] of nilpotent manifold groups, for compact orientable 3-manifolds with non-empty boundary. But we note that $M$ special does not imply $\pi_{1}(M)$ nilpotent, for example, if $\pi_{1}(M)$ is a perfect non-zero group.

In [1] the geometric approach is based on Waldhausen's hierarchies and leads to a complete classification of the 3-manifolds themselves in the more general case when $\pi_{1}(M)$ is solvable. Using the above group-theoretic approach we can simplify the geometric argument and obtain an almost homotopy-theoretic proof in the nilpotent case.

Instead of $M$ and $\widehat{M}$ we shall consider as usual the 3 -manifold $\mathcal{P}(M)=$ the Poincare associate of $M$, which is uniquely determined by $\widehat{M}=\mathcal{P}(M) \sharp \Sigma$, where $\Sigma$ is a maximal homotopy 3 -sphere and $\sharp$ denotes connected sum. Again, the fundamental groups and the boundaries of $\widehat{M}$ and $\mathcal{P}(M)$ are the same.

LEMMA 1. Let $M$ be compact, orientable such that $\mathcal{P}(M)$ has non-empty boundary. If $M$ is special then $\pi_{1}(M)$ is torsion-free and $\mathcal{P}(M)$ is a $K(\pi, 1)$-space.

Proof: Since $M$ is special, $\pi_{1}(M)$ is not a non-trivial free product. Then $\mathcal{P}(M)$ is prime [3, p.28]. Since $\partial \mathcal{P}(M) \neq \emptyset$, it follows that $\mathcal{P}(M)$ is irreducible and is in fact a $K(\pi, 1)$. This and the fact that $\mathcal{P}(M)$ is a finite-dimensional $C W$-complex implies $\pi_{1}(M)$ is torsion-free $[3, \mathrm{p} .76]$.

The following lemma is proved by a standard Van Kampen argument [1]:

LEMMA 2. If $\partial \widehat{M}$ is compressible and $\pi_{1}(M)$ is nilpotent then $\mathcal{P}(\mathcal{M})$ is homeomorphic with $S^{1} \times D$, the full torus.

CoRollary. If $M$ is a compact, oriented, 3-manifold such that $\partial \mathcal{P}(M) \neq \emptyset$ and if $\pi_{1}(M)$ is nilpotent then $\mathcal{P}(M)$ is homeomorphic to $D \times S^{1}$ or $S^{1} \times S^{1} \times I$.

PRoOF: By Theorem 1 and Lemma $1, \pi_{1}(M)=\mathbf{Z}$ or $\pi_{1}(M)=\mathbf{Z} \oplus \mathbf{Z}$. Again let $M=\mathcal{P}(M)$.

If $\pi_{1}(M)=\mathbf{Z}$ then $\partial M$ is compressible since $\operatorname{Ker}\left(\pi_{1}(\partial M) \longrightarrow \pi_{1}(M)\right) \neq 0$ and by the loop theorem there exists a compressing disk. By Lemma $2, M=S^{1} \times D$.

If $\pi_{1}(M)=\mathbf{Z} \oplus \mathbf{Z}$, by Stallings' theorem $\mathcal{P}(M)$ fibers over $S^{1}$ with fiber $F$, a closed connected 2-sided surface with boundary such that $\pi_{1}(F)=\mathbf{Z}$. Therefore $F$ is an annulus $S^{1} \times I$ and by orientability $\mathcal{P}(M)=S^{1} \times S^{1} \times I$.

On the other hand, Theorem 1 shows that special 3-manifolds with non-empty boundary have formal one-minimal models, that is, that they are determined from the cohomology only. By [2], closed 3-manifolds are not formal, because the Heisenberg groups $H_{n}$ with $n \neq 0$ are not formal. 


\section{REFERENCES}

[1] B. Evans, L. Moser, 'Solvable fundamental groups of compact 3-manifolds', Trans. Amer. Math. Soc. 168 (1972), 189-210.

[2] M. Freedman, R. Hain, P. Teichner, 'Betti number estimates for nilpotent groups', in Fields medalists' lectures, (M. Atiyah et al., Editors), World Sci. Series in 20-th Century Math. 5, 1997.

[3] J. Hempel, 3-manifolds (Princeton Univ. Press, Princeton, N.J., 1976).

[4] R. Stong, 'Quotients of the lower central series of finitely generated groups', (preprint).

[5] D. Sullivan, 'Infinitesimal computations in topology', Publ. Math. I.H.E.S. 47 (1977), 269-331.

[6] V.G. Turaev, 'Nilpotent homotopy types of closed 3-manifolds', in Proceedings of a Topology Conference in Leningrad, Springer Lecture Notes in Math 1060 (Springer-Verlag, Berlin, Heidelberg, New York, 1982), pp. 355-366.

Department of Mathematics

University of California at San Diego

La Jolla, CA 92037

United States of America

Current address:

Department of Mathematics

University of California

Santa Barbara, CA 93106

United States of America 